\title{
Enhancing the efficiency of the Pichia pastoris $A O X 1$ promoter via the synthetic positive feedback circuit of transcription factor Mxr1
}

Ching-Hsiang Chang, Hao-An Hsiung, Kai-Lin Hong and Ching-Tsan Huang ${ }^{*}$ (D)

\begin{abstract}
Background: The methanol-regulated $A O X 1$ promoter $\left(\mathrm{P}_{A O X_{1}}\right)$ is the most widely used promoter in the production of recombinant proteins in the methylotrophic yeast Pichia pastoris. However, as the tight regulation and methanol dependence of $\mathrm{P}_{A O X_{1}}$ restricts its application, it is necessary to develop a flexible induction system to avoid the problems of methanol without losing the advantages of $\mathrm{P}_{A O X 1}$. The availability of synthetic biology tools enables researchers to reprogram the cellular behaviour of $P$. pastoris to achieve this goal.

Results: The characteristics of $\mathrm{P}_{A O X_{1}}$ are highly related to the expression profile of methanol expression regulator 1 (Mxr1). In this study, we applied a biologically inspired strategy to reprogram regulatory networks in $P$. pastoris. A reprogrammed P. pastoris was constructed by inserting a synthetic positive feedback circuit of Mxr1 driven by a weak AOX2 promoter $\left(\mathrm{P}_{A O X_{2}}\right)$. This novel approach enhanced $\mathrm{P}_{\mathrm{AOX} 1}$ efficiency by providing extra Mxr1 and generated switchable Mxr1 expression to allow $P_{A O X}$ to be induced under glycerol starvation or carbon-free conditions. Additionally, the inhibitory effect of glycerol on $\mathrm{P}_{\mathrm{AOX} 1}$ was retained because the synthetic circuit was not activated in response to glycerol. Using green fluorescent protein as a demonstration, this reprogrammed $P$. pastoris strain displayed stronger fluorescence intensity than non-reprogrammed cells under both methanol induction and glycerol starvation. Moreover, with single-chain variable fragment (scFv) as the model protein, increases in extracellular scFv productivity of 98 and 269\% were observed in Mxr1-reprogrammed cells under methanol induction and glycerol starvation, respectively, compared to productivity in non-reprogrammed cells under methanol induction.
\end{abstract}

Conclusions: We successfully demonstrate that the synthetic positive feedback circuit of Mxr1 enhances recombinant protein production efficiency in P. pastoris and create a methanol-free induction system to eliminate the potential risks of methanol.

Keywords: Pichia pastoris, Recombinant protein expression, AOX1 promoter, Methanol, Methanol expression regulator 1, Synthetic gene circuit, Transcriptional reprogramming

\footnotetext{
* Correspondence: cthuang@ntu.edu.tw

Department of Biochemical Science and Technology, National Taiwan

University, No. 1, Sec. 4, Roosevelt Road, Taipei 10617, Taiwan
}

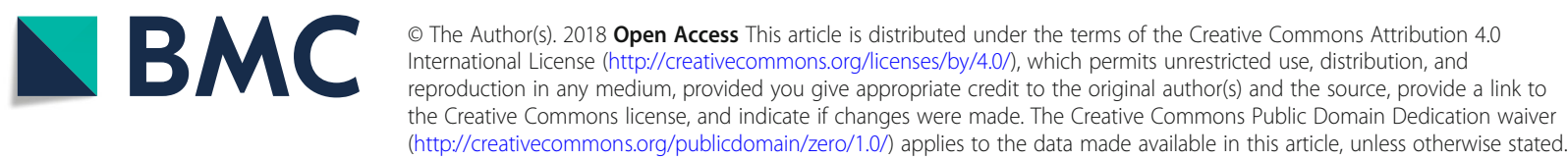




\section{Background}

The methylotrophic yeast Pichia pastoris has been extensively used in the production of recombinant proteins because it provides the advantages of post-translational modification in a eukaryotic single-cell system. Protein production in $P$. pastoris is typically driven by the $A O X 1$ promoter $\left(\mathrm{P}_{A O X I}\right)$, which occurs in response to methanol induction due to its strong and regulatable characteristics $[1,2]$. To date, more than 5000 recombinant proteins have been successfully produced in P. pastoris [3, 4]. Despite the potential of the $P$. pastoris expression system, tightly regulated $\mathrm{P}_{A O X 1}$ limits expression to restrictive conditions, with the presence of repressing carbon sources significantly decreasing recombinant protein expression during the methanol-induction phase [5]. Residual carbon sources can be removed by medium replacement prior to methanol induction, though this process is not applicable in large-scale production [6]. In addition, methanol is a toxic and flammable compound that presents some potential problems as the inducer or carbon source [7-11].

The development of synthetic biology tools has enabled researchers to reprogram cellular behaviour in P. pastoris to avoid the drawbacks of tight $\mathrm{P}_{A O X I}$ regulation. The use of alternative promoters or depressed $\mathrm{P}_{A O X 1}$ variants both provided solutions for methanol-independent production [12-14]. The improvement of induction efficiency of $\mathrm{P}_{A O X 1}$ under non-methanol carbon sources was achieved by reprogramming the carbon metabolic pathway of $P$. pastoris $[15,16]$. However, such a strategy might interrupt carbon metabolism and result in growth defects. An alternative approach is to reprogram transcriptional regulation of $\mathrm{P}_{A O X 1}$. Although the regulation mechanism of $\mathrm{P}_{A O X 1}$ is not fully understood, several transcription factors involved in $\mathrm{P}_{A O X 1}$ regulation have been identified. In response to different carbon sources, $\mathrm{P}_{A O X 1}$ has three regulated stages of gene expression including repression, derepression, and activation [17]. Among carbon regulation of $\mathrm{P}_{A O X 1}$, the transcriptional activator Mxr1 is constitutively expressed and plays a crucial role in $\mathrm{P}_{A O X 1}$ derepression and activation processes [18-20]. The Nrg1 repressor participates in the inhibition mechanism by competing for Mxr1 binding elements in $\mathrm{P}_{A O X 1}$ [21]; Nrg1 can be down-regulated by switching the carbon source from glycerol to methanol [22], though the detailed expression pattern remains unclear. During the activation process, the activators Prm1 and Mit1 are up-regulated by methanol to activate $\mathrm{P}_{A O X 1}$ expression $[19,20]$. In previous studies, the $\mathrm{P}_{A O X 1^{-}}$-based methanol free expression system could be achieved by deletion of three repressors (Nrg1, Mig1 and Mig2) and overexpression of one activator (Mit1) [3], or by derepressed overexpression of Mxr1 or Mit1 [23]. However, NRG1 deletion might lead to the potential risk of growth defects [21]. Hence, developing the synthetic circuits to provide an efficient approach for controlling the characteristics of $\mathrm{P}_{A O X 1}$, remains a challenge.
In this study, we reprogrammed regulatory networks in $P$. pastoris through biological inspiration of another methylotrophic yeast: Hansenula polymorpha. Unlike $\mathrm{P}_{A O X 1}$ in P. pastoris, glycerol does not interfere with the methanol-induced efficiency of $\mathrm{P}_{M O X}$, the alcohol oxidase promoter in $H$. polymorpha. In addition to induction by methanol, $\mathrm{P}_{M O X}$ can express recombinant genes via a carbon starvation strategy $[17,24]$. The regulatory difference between $\mathrm{P}_{A O X 1}$ and $\mathrm{P}_{M O X}$ results from upstream transcriptional networks in cells rather than due to promoter sequences alone [25]. Interestingly, this phenomenon might be related to the different expression pattern between P. pastoris Mxr1 and its orthologous gene (HPODL00650) in H. polymorpha, as HPODL00650 is up-regulated by methanol [26]. We speculate the existence of positive feedback regulation of HPODL00650 in $H$. polymorpha, which might contribute to the flexible activation of $\mathrm{P}_{M O X 1}$. Therefore, to mimic the expression pattern of HPODL00650, a reprogrammed $P$. pastoris strain was constructed by inserting a synthetic positive autoregulation circuit of Mxr1. In addition to endogenous Mxr1, exogenous Mxr1 was driven by the methanol-regulated $A O X 2$ promoter $\left(\mathrm{P}_{A O X 2}\right)$, a promoter that is weaker than $\mathrm{P}_{A O X 1}$. This novel strategy did not affect cells in the repression condition, thus maintaining tight regulation of $\mathrm{P}_{A O X 1}$ and preventing growth defects. We demonstrate herein that the transcriptional efficiency of $\mathrm{P}_{A O X I}$ was enhanced and that the interference due to residual repressing carbon sources was reduced. These Mxr1-reprogrammed cells show great potential for broader applications.

\section{Results}

Mxr1-reprogrammed cells had altered GFP expression but did not show growth defects

Mxr1-reprogrammed cells were constructed by exogenous expression of Mxr1 controlled by the $\mathrm{P}_{A O X 2}$ in a clone carrying nine copies of the $\mathrm{P}_{A O X 1}$-regulated GFP expression cassette. Non-reprogrammed $P$. pastoris KM71/GFP and reprogrammed $P$. pastoris KM71m/GFP were compared to evaluate the effects of the synthetic Mxr1 circuit on cell growth and heterologous gene expression. Figure 1 illustrates that $P$. pastoris KM71m/GFP cells exhibited stronger fluorescence intensity than did $P$. pastoris KM71/GFP cells during methanol induction. In addition, no significant differences in cell growth were found throughout the experiments, suggesting that expression of Mxr1 by the mild $\mathrm{P}_{A O X 2}$ promoter did not cause growth defects. However, as evidenced by GFP production prior to methanol induction, an altered Mxr1 expression pattern might result in the potential risk of $\mathrm{P}_{A O X I}$ leakage. 


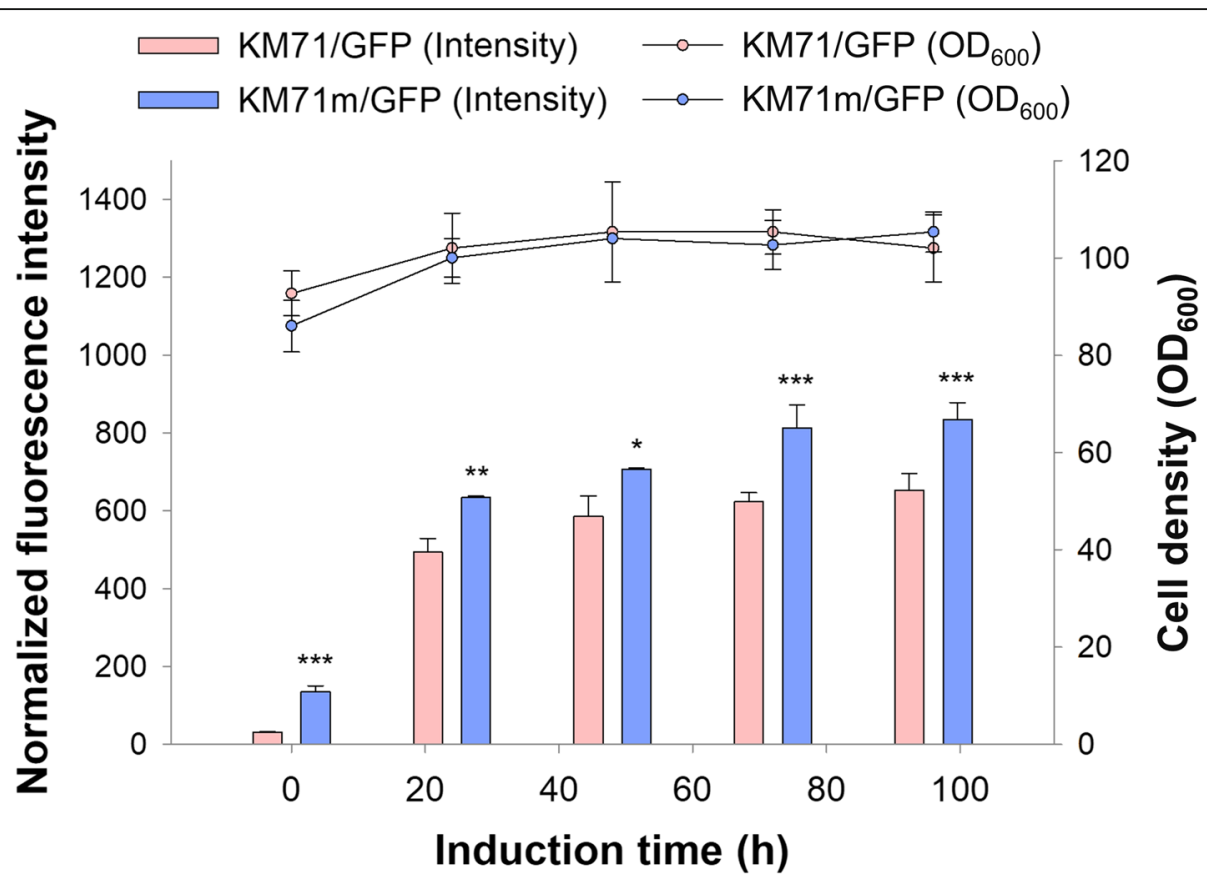

Fig. 1 Mxr1-reprogrammed cells had altered GFP expression but did not show growth defects. After being cultured in BMGY (1\% glycerol), cells were cultured in BMMY and induced with $0.5 \%$ methanol daily. Cell density $\left(\mathrm{OD}_{600}\right)$ is presented by a line and scatter plot, and the normalized fluorescence intensity is presented by a bar plot. Error bars represent the standard deviation of three biological replicates. The independentsample t-test was used to determine significance. ${ }^{*}, p<0.05 ;{ }^{* *}, p<0.01 ;{ }^{* *}, p<0.005$

$\mathrm{P}_{\text {AOX1 } 1}$ remained controllable in Mxr1-reprogrammed cells Several studies have suggested that $\mathrm{P}_{A O X 1}$ was leaky under derepression conditions, especially in bioreactor cultivations [27-29]. Therefore, to assess the level of $\mathrm{P}_{A O X 1}$ leakage, cells were cultured in different concentrations of glycerol for $24 \mathrm{~h}$. As shown in Fig. 2a, the cell density $\left(\mathrm{OD}_{600}\right)$ of both $P$. pastoris KM71/GFP and P. pastoris KM71m/GFP cell cultures increased with a rise in glycerol concentration, indicating that the carbon source was the limiting factor in a 24-h culture; furthermore, no growth
A

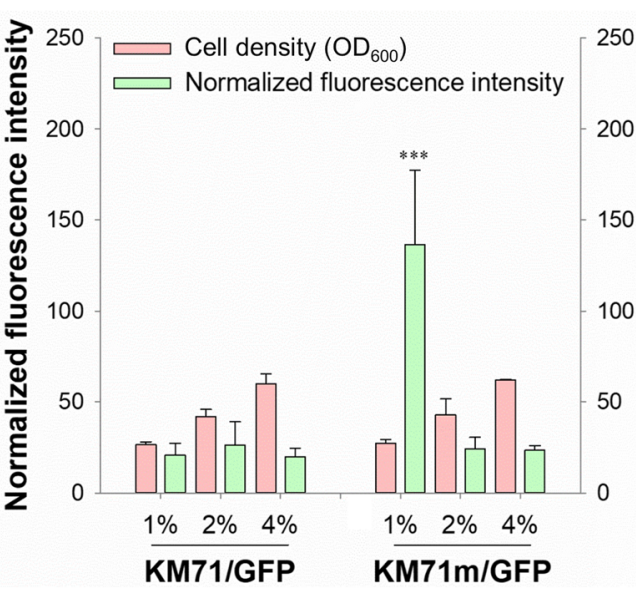

B

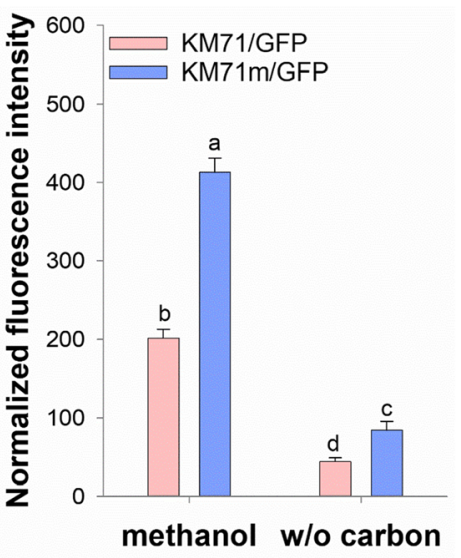

Fig. $2 P_{A O X}$ remained controllable in Mxr1-reprogrammed cells. a Detection of the cell density $\left(\mathrm{OD}_{600}\right)$ and normalized fluorescence intensity of cells grown in different concentrations of glycerol for $24 \mathrm{~h}$. The error bars represent the standard deviation of three biological replicates. Two-way analysis of variance (ANOVA) and the Tukey test were used to determine significance. ${ }^{* * *}, p<0.005$. b After being cultured in BMGY ( $2 \%$ glycerol), the cells were cultured in BMNY or BMMY. Detection of the normalized fluorescence intensity of cells grown in different carbon sources for $3 \mathrm{~h}$. The error bars represent the standard deviation of three biological replicates. Two-way ANOVA and the Tukey test were used to determine significance. Groups with different letters are significantly different 
defects of the reprogrammed cells were found. As expected, only the baseline fluorescence intensity was observed for P. pastoris KM71/GFP cells under 1, 2 and $4 \%$ glycerol. In contrast, the fluorescence intensity of $P$. pastoris $\mathrm{KM} 71 \mathrm{~m} / \mathrm{GFP}$ cells significantly increased under conditions of $1 \%$ glycerol, though no significant difference in $P$. pastoris KM71/GFP cells under 2 or $4 \%$ glycerol was found. Based on these results, it was plausible that $\mathrm{P}_{A O X 1}$ in $P$. pastoris $\mathrm{KM} 71 \mathrm{~m} / \mathrm{GFP}$ cells was activated by glycerol depletion. To verify this assumption, a glycerol-depletion condition was established by replacing $2 \%$ glycerol with a carbon-free medium. As shown in Fig. 2b, P. pastoris KM71m/GFP cells displayed enhanced GFP expression, regardless of whether they were induced by methanol. Compared with $P$. pastoris KM71/GFP under methanol induction, the relative GFP production by $P$. pastoris KM71m/GFP cells was $205 \pm 9$ and $42 \pm 6 \%$ under methanol induction and carbon starvation, respectively. These results suggested that reprograming Mxr1 improved protein production efficiency and resulted in flexible activation conditions without interfering with the controllable characteristics of $\mathrm{P}_{A O X 1}$.

\section{Positive feedback of Mxr1 increased the transcriptional efficiency of $\mathrm{P}_{A O X 1}$ and broke the Mxr1 titration effect}

To further confirm that the increase in GFP expression resulted from enhanced transcriptional efficiency, mRNA expression levels of GFP and Mxr1 were determined by quantitative reverse transcription-polymerase chain reaction (RT-qPCR). In the presence of glycerol, mRNA expression levels of GFP in both reprogrammed and non-reprogrammed cells were repressed, with no significant difference. In contrast, the transcriptional efficiency of $\mathrm{P}_{A O X 1}$ in $P$. pastoris $\mathrm{KM} 71 \mathrm{~m} / \mathrm{GFP}$ cells was significantly increased in response to methanol induction or carbon starvation (Fig. 3a). As shown in Fig. 3b, Mxr1 was, not surprisingly, constitutively expressed in P. pastoris KM71/GFP, and equal Mxr1 expression was detected in $P$. pastoris KM71m/GFP cells in response to glycerol. However, the expression levels of Mxr1 showed 13.9-fold and 35.4-fold increases in $P$. pastoris $\mathrm{KM} 71 \mathrm{~m} / \mathrm{GFP}$ cells under carbon starvation and methanol induction, respectively. Although Mxr1 expression showed higher fold increases compared to GFP after reprogramming, the transcript levels of Mxr1 were lower than those of GFP due to the weak $\mathrm{P}_{A O X 2}(-$ Additional file 1: Figure S1). These results suggested that the positive feedback circuit of Mxr1 enhanced $\mathrm{P}_{A O X 1}$ transcriptional efficiency without breaking the inhibitory effect of glycerol.

According to the study of Camara et al., the Mxr1 titration effect is a plausible explanation for the observed transcriptional attenuation of methanol-induced genes in increasingly used $\mathrm{P}_{A O X I^{-}}$-regulated expression cassettes [30]. To verify whether this Mxr1 titration effect can be disrupted by exogenous expression of Mxr1, the methanol utilization capacities of $P$. pastoris $\mathrm{KM} 71 \mathrm{H}, P$. pastoris KM71/GFP, and P. pastoris KM71m/GFP cells were determined (Fig. 3c). As evidenced by a colorimetric assay, AOX activity in $P$. pastoris KM71/GFP cells was weaker than that in $P$. pastoris $\mathrm{KM} 71 \mathrm{H}$ cells in response to methanol. However, the defect in methanol utilization was recovered in P. pastoris KM71m/GFP cells, suggesting that the expression level of Mxr1 was a bottleneck for heterologous gene expression. Hence, breaking the Mxr1 titration effect by a synthetic Mxr1 circuit is expected to increase the potential of using a high copy-number strategy.
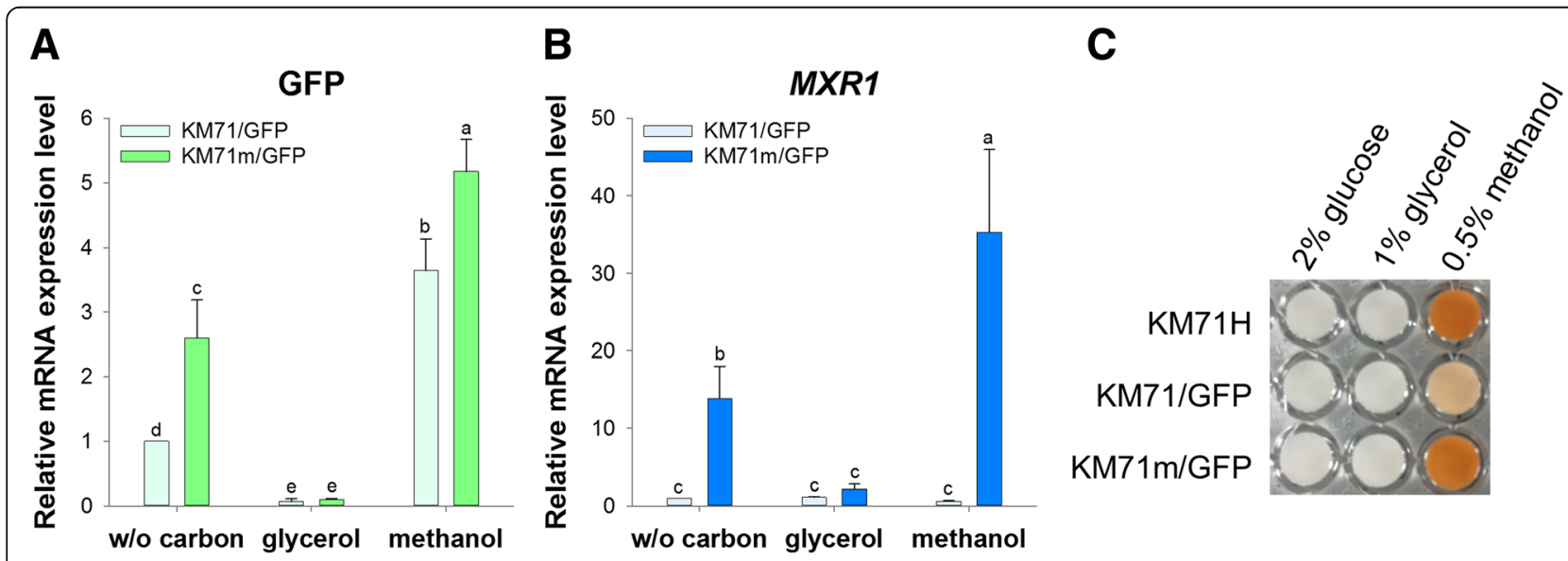

Fig. 3 Positive feedback of Mxr1 increased the transcriptional efficiency of $P_{A O X 1}$ and broke the Mxr1 titration effect. mRNA was extracted from cells cultured in different carbon sources for $3 \mathrm{~h}$. a GFP. b MXR1. mRNA levels in each sample were normalized to $18 \mathrm{~S}$ rRNA. The relative expression level for each gene was normalized to the control grown under the carbon-free condition. The error bars represent the standard deviation of three biological replicates. Two-way ANOVA and the Tukey test were used to determine significance. Groups with different letters are significantly different. c AOX activity in different cells in response to different carbon sources 


\section{Mxr1-reprogrammed cells were inducible under non- restricted conditions}

Figure 4a presents the AOX activity of P. pastoris KM71/ GFP and $P$. pastoris $\mathrm{KM} 71 \mathrm{~m} / \mathrm{GFP}$ cells in response to different carbon combinations. The tight $\mathrm{P}_{A O X 1}$ regulation of $P$. pastoris KM71/GFP cells resulted in no AOX activity under glycerol and carbon-free conditions, and methanolinduction efficiency was also interfered by low concentration of glycerol. Conversely, P. pastoris KM71m/GFP cells not only bypassed the interference caused by small amount of glycerol but also were induced under the carbon-free condition. These results suggested that the flexible characteristic of $\mathrm{P}_{M O X}$ was achieved by reprogramming the regulation pattern of Mxr1.

Although the fluorescence intensity of $P$. pastoris KM71/GFP was enhanced by medium replacement (Fig. 4b), the intensity of P. pastoris KM71m/GFP with or without medium replacement was significantly higher than that of $P$. pastoris KM71/GFP with medium replacement. These results suggested that Mxr1 reprogramming overcame the interference of residual repressive carbon and resulted in a smooth transition between glycerol and methanol. In addition to the above advantage, Mxr1 reprogramming showed great potential in the development of a methanol-free induction system. As shown in Fig. 4c, the fluorescence intensity of $P$. pastoris $\mathrm{KM} 71 \mathrm{~m} / \mathrm{GFP}$ increased significantly by the daily addition of $0.33 \%$ glycerol, whereas only baseline intensity was detected for $P$. pastoris KM71/GFP. These results indicated that Mxr1-reprogrammed $P$. pastoris is inducible under glycerol depletion condition.

\section{Application of Mxr1-reprogrammed cells in the production of scFv}

To demonstrate the application feasibility of Mxr1reprogramming strategy in the production of functional recombinant proteins, a secreted single-chain variable fragment $(\mathrm{scFv})$ production strain which contain one copy of $\mathrm{P}_{A O X 1^{-}}$-scFv cassette was transformed with linearized Mxr1 reprogrammed plasmid or empty vector. The results of comparison of secreted $\mathrm{scFv}$ production between reprogrammed cells $\mathrm{KM} 71 \mathrm{Hm} / \mathrm{scFv}$ and non-reprogrammed cells $\mathrm{KM} 71 \mathrm{H} / \mathrm{scFv}$ under methanol induction or glycerol starvation are shown in Fig. 5a. Compared to scFv production in non-reprogrammed cells under methanol induction, $\mathrm{scFv}$ expression titre in $P$. pastoris $\mathrm{KM} 71 \mathrm{Hm} / \mathrm{scFv}$ showed a $98 \pm 28 \%$ increase under methanol induction and a $269 \pm 28 \%$ increase under glycerol starvation. These results suggested that the Mxr1-reprogramming strategy show same effects in single copy strain. Notably, western blot analysis revealed that a large amount of $\mathrm{scFv}$ remained within
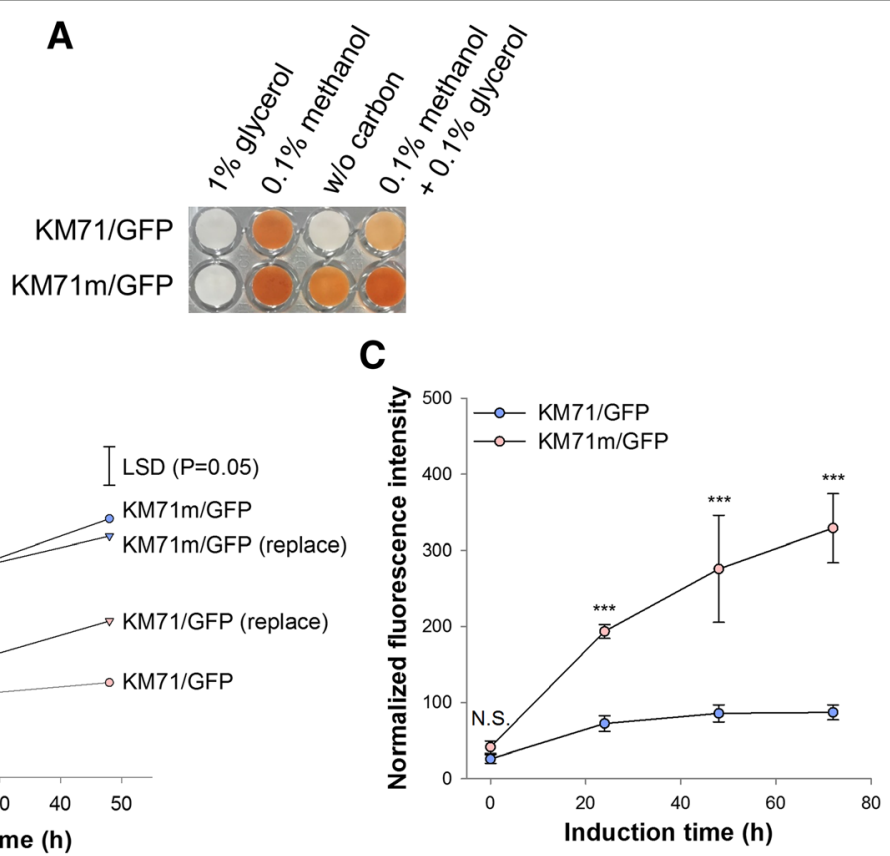

Fig. 4 Mxr1-reprogrammed cells were induced under broad conditions. a AOX activity in different cells in response to different carbon sources. b After being cultured in BMGY ( $2 \%$ glycerol), cells were concentrated in the original medium, or the medium was replaced by fresh medium without residual glycerol. The cells were then induced with $0.5 \%$ methanol per day. The results represent the mean of three independent biological replicates. Two-way ANOVA and the least significant difference (LSD) test were used to determine significance $(p=0.05)$. c After being cultured in BMGY (2\% glycerol), cells were cultured in BMGY and induced with $0.33 \%$ glycerol per day. The error bars represent the standard deviation of three biological replicates. The independent-sample t-test was used to determine significance. NS, $p>0.05 ;{ }^{* *}, p<0.005$ 
A

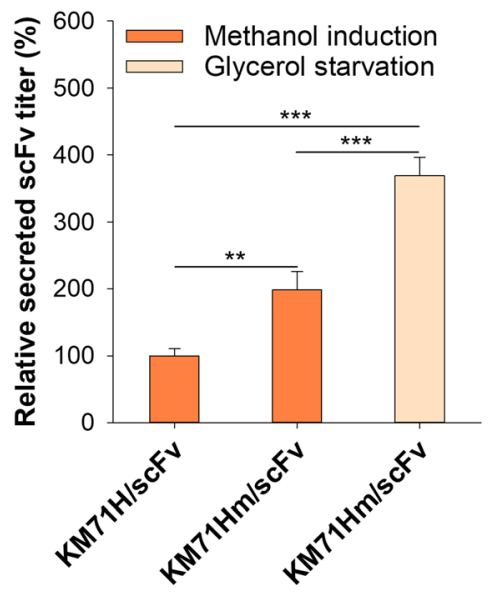

B

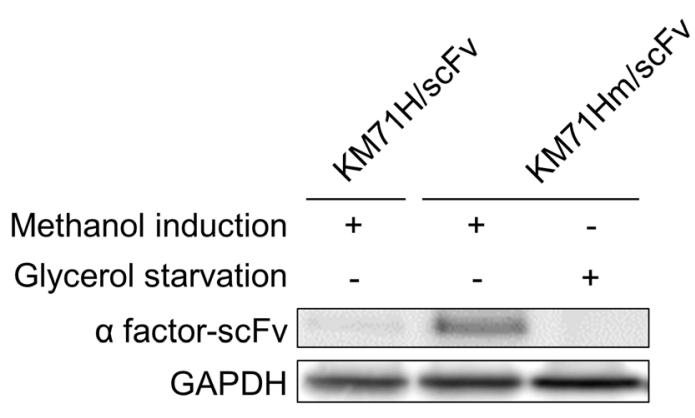

Fig. 5 Synthetic circuit of Mxr1 enhanced scFv expression. After being cultured in BMGY (1\% glycerol), cells were cultured in BMMY and induced with $1 \%$ methanol daily. a The relative secreted scFv titre is presented. The error bars represent the standard deviation of three biological replicates. One-way ANOVA and the Tukey test were used to determine significance. ${ }^{*}, p<0.05 ;{ }^{* *}, p<0.005$. b Intracellular proteins after induction were examined by western blotting using an anti-his antibody. GAPDH was used as the loading control

P. pastoris $\mathrm{KM} 71 \mathrm{Hm} / \mathrm{scFv}$ under methanol induction, though only a trivial amount was detected under glycerol starvation (Fig. 5b). This accumulation of intracellular $\mathrm{scFv}$ in the reprogrammed $P$. pastoris cells suggested that the potential of Mxr1-reprograming was not fully exhibited, as secretion capacity might be the limiting factor in the production of extracellular recombinant proteins. Although further efforts are still required to resolve the observed secretion limitation in methanol-induced Mxr1-reprogrammed P. pastoris, recombinant protein production in reprogrammed $P$. pastoris under glycerol starvation appeared to be efficient and applicable.

\section{Discussion}

Based on previous studies and the results of this study, we propose a putative mechanism of $\mathrm{P}_{A O X 1}$ transcriptional regulation in reprogrammed $P$. pastoris, as shown in Fig. 6. In this scheme, the exogenous Mxr1 expressed by $\mathrm{P}_{A O X 2}$ establishes a positive auto-regulation circuit in

\section{Reprogrammed Cells}

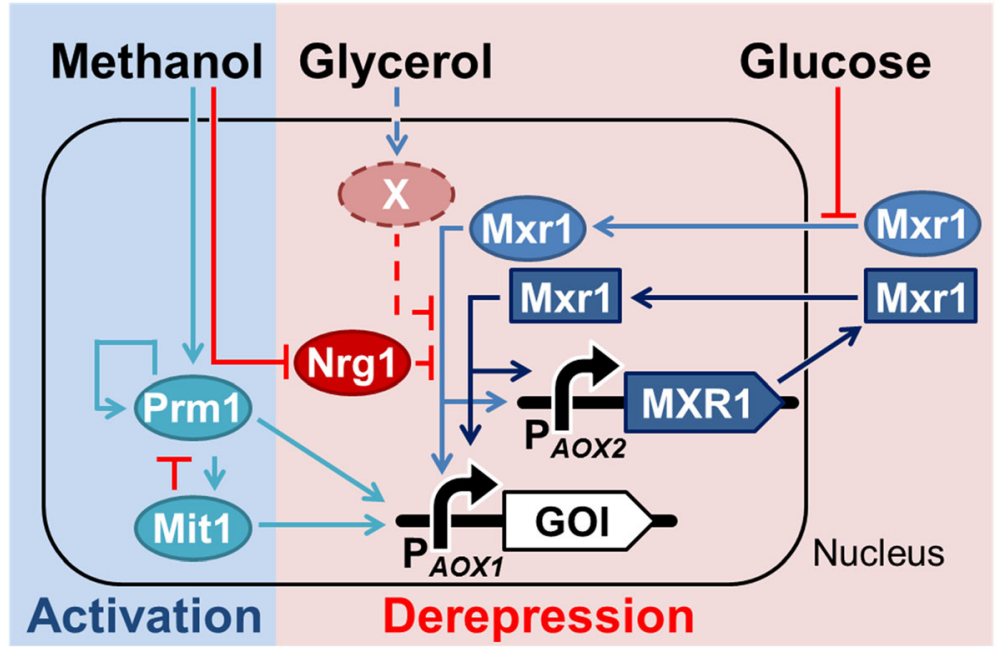

Fig. 6 The schematic diagram of transcription regulation in Mxr1-reprogrammed cells. There are two phases, derepression and activation, for $P_{\text {AOx }}$ activation in P. pastoris. Prm1, Mit1, and Mxr1 are activators (blue). Nrg1 and X are repressors (red). The dashed line and repressor X are speculated based on our results, for which further investigation is needed. In reprogrammed cells, we used $P_{\text {AOx2 }}$ to express extra Mxr1 (dark blue square) 
reprogrammed $P$. pastoris cells. When the medium contains glycerol, endogenous Mxr1 enters the nucleus but does not activate $\mathrm{P}_{A O X 1}$ due to the presence of Nrg1 and other repressors [18, 21, 31]. Moreover, the synthetic $\mathrm{P}_{A O X 2}$-regulated circuit is not activated under glycerol repression, and hence reprogrammed $P$. pastoris cells do not exhibit leaky expression or growth defects. When the medium contains methanol, the synthetic Mxr1-reprogrammed circuit is activated in addition to the endogenous regulation machinery $[19,20,22]$. The increased amount of Mxr1 further improves the methanol-induced transcriptional efficiency of $\mathrm{P}_{A O X 1}$ and breaks the limitation of the Mxr1 titration effect [30]. In our study, these is the reason why heterologous gene expression in reprogrammed $P$. pastoris was higher than that in the non-reprogrammed counterpart under methanol induction. In contrast, small amount of residual glycerol did not interfere with the methanol-induced efficiency of $\mathrm{P}_{A O X 1}$ in Mxr1-reprogramed cells, allowing a smooth transition between glycerol and methanol. Besides the plausible explanation that endogenous repression threshold is overcome by the added Mxr1, Zhan et al. indicated that overexpression of Mxr1 represses the expression of glycerol transporter 1 ( $g t 1)$ by binding to the promoter of $g t 1$ $\left(\mathrm{P}_{G T 1}\right)$, consequently reducing the content of glycerol in cells and promoting the activation of $\mathrm{P}_{A O X 1}$ [32].

In addition to Mxr1, the role of the repressor Nrg1 was also investigated (Additional file 2: Figure S2). The expression level of Nrg1 was down-regulated in response to methanol. However, there was no significant difference in Nrg1 expression levels between the glycerol group and the no-carbon-source group. It was interesting that the engineered cells could reach high Mxr1 expression level in carbon-free medium without down-regulation of Nrg1. Hence, we speculate the existence of repressor X, which is regulated by glycerol. Under glycerol-starvation conditions, down-regulation of repressor $\mathrm{X}$ has a lower inhibitory effect on $\mathrm{P}_{A O X 1}$; however, $\mathrm{P}_{A O X 1}$ is still repressed in non-reprogrammed cells due to weak constitutive expression of endogenous Mxr1. In contrast, down-regulation of repressor $\mathrm{X}$ would generate the different strength of repression between glycerol repression and derepression conditions, which explains why $\mathrm{P}_{A O X 1}$ in reprogrammed cells was only activated in glycerol depletion condition but not in glycerol repression condition. Repressor X is likely to be Mig1 or Mig2, as their response to glycerol or methanol is consistent with that of repressor $\mathrm{X}[3,22]$. Regardless, the detailed expression patterns of Mig1 and Mig2 under glycerol, methanol and carbon depletion require further investigation.

Overexpression or deletion of transcription factors is the simplest way to control $\mathrm{P}_{A O X 1}$ expression; however, these strategies might result in cellular growth defects or break the tight regulation of $\mathrm{P}_{A O X 1}$ in response to repressive carbon sources [23, 32, 33]. Recently, Vogl et al. converted a $\mathrm{P}_{A O X 1}$-based expression strain into a methanol-free production system without breaking the inhibitory effect of glucose through derepressed overexpression of Mxr1 or Mit1 under CAT1 promoter regulation [23]. Interestingly, a methanol-free production system was also achieved in our study by generating switchable Mxr1 expression via a synthetic positive feedback circuit, even though $\mathrm{P}_{A O X 2}$ is not a naturally derepressed promoter $[17,34]$. Compared with the derepressed overexpression of Mxr1, the positive feedback circuit of Mxr1 showed better improvement of productivity under methanol induction and derepression condition. Meanwhile, extra Mxr1 regulated by weak $\mathrm{P}_{A O X 2}$ could prevent the detrimental effects of strong Mxr1 expression [18]. However, both strategies indicate that a synthetic circuit resulting in different levels of transcription factor expression under various conditions is a viable and flexible approach to controlling the characteristics of $\mathrm{P}_{A O X 1}$. Although only the Mut ${ }^{\mathrm{S}}$ strain was used in this study, Vogl et al. reported that the Mut phenotype was independent to the methanol regulation machinery [23]. The strategy of transcriptional reprogramming is expected to be effective on both $\mathrm{Mut}^{+}$and $\mathrm{Mut}^{\mathrm{S}}$ strains.

In addition to the transcriptional process, the secretion pathway is also a bottleneck of recombinant protein production in $P$. pastoris $[35,36]$. Previous studies have shown that using a weak promoter could lower the production rate of recombinant proteins, which was favorable for the secretion of proteins with complex folding [14]. Mxr1-reprogrammed P. pastoris with the glycerol starvation strategy could achieve the similar goal of slowing down the expression level and get a better productivity of secreted protein. On the other hand, co-expression of target proteins with chaperone protein Kar2 [37], protein disulphide isomerase (PDI) or transcription factors such as Aft1 [38] and Hac1 [39] could enhance the productivity of certain proteins in P. pastoris [40]. Hence, the combination of Mxr1 reprogramming with other secretion-related gene circuits is necessary to solving the problem of intracellular target protein accumulation.

\section{Conclusions}

We demonstrated that the recombinant protein production driven by $\mathrm{P}_{A O X 1}$ was greatly enhanced by the synthetic positive feedback circuit of Mxr1 in P. pastoris under methanol induction. Breaking the Mxr1 titration effect by added Mxr1 increased the potential of using a high copy-number strategy. In addition, Mxr1 reprogramming reduced interference from the residual repressing carbon source, allowing a smooth transition between different carbon sources. This platform also provided an alternative approach to expressing target genes driven by $\mathrm{P}_{A O X 1}$ under glycerol starvation, thereby 
eliminating the potential risks of methanol. These Mxr1reprogrammed cells are expected to have great potential for broader applications.

\section{Methods}

\section{Plasmids and strains}

Standard procedures for the construction of plasmid DNA and transformation of $P$. pastoris were described previously [41]. GFP production cells were first constructed by expressing GFP controlled by the $A O X 1$ promoter in the $\mathrm{Mut}^{\mathrm{S}}$ strain $P$. pastoris KM71. Mxr1-reprogrammed cells were then constructed by exogenous expression of Mxr1 controlled by the $A O X 2$ promoter in a clone carrying nine copies of the GFP expression cassette. Empty vector control cells were constructed to restore the histidine deficiency of the GFP production clone. In both the empty vector control and Mxr1-reprogrammed cells, clones with only one copy of the $\mathrm{P}_{A O X 2}$-regulated cassette were selected for further analysis and named KM71/GFP and KM71m/GFP, respectively. Single-chain variable fragment $(\mathrm{scFv})$ production cells were obtained by transforming pPICZ $\alpha \mathrm{A}-\mathrm{scFv}$ into $P$. pastoris $\mathrm{KM} 71 \mathrm{H}$. The pAOX2KH and pAOX2KH-Mxr1p plasmids were transformed separately into the clone carrying one copy of the scFv expression cassette to generate the empty vector control and Mxr1-reprogrammed cells, which were named KM71H/ $\mathrm{scFv}$ and $\mathrm{KM} 71 \mathrm{Hm} / \mathrm{scFv}$, respectively. The strains used in this study and their characteristics are listed in Table 1. The primers used for plasmids construction and sequencing are provided in Additional file 3: Table S1.

\section{Media and culture conditions}

The media used in this study are listed in Additional file 4: Table S2. The protein expression procedure for P. pastoris was conducted according to the manufacturer's instructions (Invitrogen, Carlsbad, California, USA). Cells were cultured in $3 \mathrm{~mL}$ YPDZ for $20 \mathrm{~h}$ as seed culture and inoculated into $100 \mathrm{~mL}$ BMGY medium to an optical density at $600 \mathrm{~nm}\left(\mathrm{OD}_{600}\right)$ of 0.15 . The cells were grown at $30^{\circ} \mathrm{C}$, $250 \mathrm{rpm}$ for $24 \mathrm{~h}$ and harvested by centrifugation at
$3000 \times g$ for $10 \mathrm{~min}$ to remove residual repressive carbon sources in the supernatant. The pellet was resuspended in $20 \mathrm{~mL}$ of a different carbon source medium, BMMY, BMGY, or BMNY. BMGY was a fresh medium with a different concentration of glycerol. BMMY was a fresh medium with $0.5 \%$ methanol added every $24 \mathrm{~h}$ to induce protein expression. BMNY was a fresh medium without a carbon source.

\section{Protein expression analysis}

Quantification of GFP expression was monitored using a SpectraMax M2e Microplate Reader (Molecular Device, Sunnyvale, California, USA). The excitation wavelength was $488 \mathrm{~nm}$, with an emission wavelength of $509 \mathrm{~nm}$. The GFP expression level was normalized to the cell density, which was monitored by optical density at $600 \mathrm{~nm}$. Extracellular expression of $\mathrm{scFv}$ was analysed by sodium dodecyl sulphate polyacrylamide gel electrophoresis (SDS-PAGE), followed by Coomassie Brilliant Blue G-250 staining. The relative $\mathrm{scFv}$ titre was determined using UVP image analysis software (Analytik Jena AG, Jena, Germany). For intracellular protein analysis, cell pellets were harvested by centrifugation and washed once with the same volume of lysis buffer $(50 \mathrm{mM}$ monosodium phosphate $\mathrm{pH} 7.4,1 \mathrm{mM}$ ethylenediaminetetraacetic acid (EDTA), 5\% glycerol, $1 \mathrm{mM}$ phenylmethylsulfonyl fluoride (PMSF)). The cell pellets were resuspended in breaking buffer and ground in liquid nitrogen. Total intercellular proteins were transferred to a polyvinylidene fluoride (PVDF) membrane (PerkinElmer, Waltham, Massachusetts, USA) after electrophoresis and detected by specific antibodies. The primary antibody was a rabbit anti-his polyclonal antibody (Bioman, New Taipei City, Taiwan), and a horseradish peroxidase (HRP)-conjugated antibody (PerkinElmer) was used as the secondary antibody. Both antibodies were diluted 5000x with gelatine-NET $(0.15 \mathrm{M} \mathrm{NaCl}, 5 \mathrm{mM}$ EDTA, $0.05 \%$ Tween in $50 \mathrm{mM}$ Tris- $\mathrm{HCl}, \mathrm{pH} 8.0$ ) before use. Colorimetric detection was performed using an enhanced chemiluminescence substrate (PerkinElmer). The total intracellular

Table 1 Strains used in this study

\begin{tabular}{|c|c|c|}
\hline Strain & Characteristics & Source \\
\hline E. coli EPI300 & Gene cloning host & Epicentre Technologies Corp, USA \\
\hline P. pastoris KM71 & Gene expression host with histidine deficiency & Invitrogen, USA \\
\hline P. pastoris $\mathrm{KM} 71 \mathrm{H}$ & Gene expression host & Invitrogen, USA \\
\hline P. pastoris KM71/GFP & $\begin{array}{l}\text { GFP expressed in P. pastoris KM71 and histidine deficiency } \\
\text { was restored by the empty PAOX2 vector }\end{array}$ & In this study \\
\hline P. pastoris KM71m/GFP & $\begin{array}{l}\text { GFP expressed in P. pastoris KM71m containing the } \\
\text { synthetic Mxr1 circuit }\end{array}$ & In this study \\
\hline P. pastoris $\mathrm{KM} 71 \mathrm{H} / \mathrm{scFv}$ & scFv expressed in $P$. pastoris $\mathrm{KM} 71 \mathrm{H}$ & In this study \\
\hline P. pastoris $\mathrm{KM} 71 \mathrm{Hm} / \mathrm{scFv}$ & $\begin{array}{l}\text { scFv expressed in P. pastoris } \mathrm{KM} 71 \mathrm{Hm} \text { containing the } \\
\text { synthetic Mxr1 circuit }\end{array}$ & In this study \\
\hline
\end{tabular}


protein content was assessed using a rabbit anti-GAPDH polyclonal antibody (GeneTex, Irvine, California, USA).

\section{RNA expression level analysis}

The mRNA expression levels of transcription factor genes and GFP were verified by real-time PCR. Total RNA derived from cells was extracted using a NautiaZ Bacteria/Fungi RNA Mini Kit (Nautia Gene, Taipei, Taiwan) in accordance with the manufacturer's procedure. Reverse transcription was performed using ARROW-Script Reverse transcriptase III with Radom hexamers (ARROWTEC, Taipei, Taiwan), and the products were used for subsequent real-time PCR performed with the StepOne ${ }^{\text {tw }}$ System (Applied Biosystems, Foster, California, USA) using $2 \mathrm{xIQ}^{2}$ SYBR Green FAST qPCR System Master Mix-HIGH ROX (Bio-Genesis Technologies, Taipei, Taiwan). The primers used for real-time PCR are listed in Additional file 5: Table S3. After the cycle threshold values $(\mathrm{CT})$ were determined, relative fold differences were calculated using the $2^{-\Delta \Delta C T}$ method with $18 \mathrm{~S}$ rRNA as the endogenous reference gene.

\section{AOX activity assay}

Cells were cultured in $3 \mathrm{~mL}$ YPDZ for $16 \mathrm{~h}$ as seed culture and inoculated into $3 \mathrm{~mL}$ BMDY, BMGY, BMMY, BMNY, or BMGMY medium to an optical density of 1at $600 \mathrm{~nm}$ $\left(\mathrm{OD}_{600}\right)$. The cells were grown at $30^{\circ} \mathrm{C}, 250 \mathrm{rpm}$ for $10 \mathrm{~h}$, and a total of $5 \times 10^{7}$ cells was harvested by centrifugation. The cell pellets were resuspended in AOX activity reagent and incubated at $30^{\circ} \mathrm{C}$ for $30 \mathrm{~min}$ [42].

\section{Additional files}

Additional file 1: Figure S1. The delta CT values of GFP (left) and MXR1 (right) expression. The mRNA was extracted from the cells cultured in different carbon sources for $3 \mathrm{~h}$. The mRNA levels were normalized to 18S rRNA in each sample and represented by delta $C$ value. The error bars represented the standard deviation of three biological replicates. (DOCX 46 kb)

Additional file 2: Figure S2. The mRNA expression level of NRG1. The mRNA was extracted from the cells cultured in different carbon sources for $3 \mathrm{~h}$. The mRNA levels were normalized to $18 \mathrm{~S}$ rRNA in each sample. The relative expression level for each gene was normalized to the control grown in the carbon-free condition. The error bars represented the standard deviation of three biological replicates. The two-way ANOVA and Turkey test were used to determine the statistical significance. The groups with different alphabet were significantly different. (DOCX 1483 kb)

Additional file 3: Table S1. The primers used for plasmids construction and sequencing. (DOCX $12 \mathrm{~kb}$ )

Additional file 4: Table S2. The media used in this study. (DOCX $12 \mathrm{~kb}$ ) Additional file 5: Table S3. The primers used for real-time PCR. (DOCX $12 \mathrm{~kb}$ )

Additional file 6: Excel file of raw data generated or analyzed during this study. (XLSX $554 \mathrm{~kb}$ )

\section{Abbreviations}

AOX: Alcohol oxidase; BMDY: Buffered dextrose-complex medium; BMGY: Buffered glycerol-complex medium; BMMY: Buffered methanolcomplex medium; BMNY: Buffered complex medium without a carbon source; GFP: green Fluorescent protein; KM71m: Mxr1-reprogrammed KM71 strains; Mxr1: Methanol expression regulator $1 \mathrm{P}_{\text {AOX }}$ Alcohol oxidase 1 promoter; scFv: Single-chain variable fragment; YPDZ: Yeast extract-peptonedextrose with zeocin

\section{Acknowledgements}

Not applicable.

\section{Funding}

This work was supported by Ministry of Science and Technology, Taiwan, ROC (MOST-105-2313-B-002-043).

\section{Availability of data and materials}

All data generated or analyzed during this study are included in this published article and its supplementary information files, including the raw data in spreadsheets (Additional file 6).

\section{Author contributions}

$\mathrm{CHC}$ and $\mathrm{CTH}$ are responsible for the project planning and experimental design. $\mathrm{CHC}$ and $\mathrm{HAH}$ constructed the strains and performed the transcriptional and expression analyses. $\mathrm{KLH}$ contributed to the experimental operation. $\mathrm{CHC}, \mathrm{KLH}$ and $\mathrm{HAH}$ analysed the results and wrote the manuscript. $\mathrm{CTH}$ revised and produced the final manuscript. All authors contributed to scientific discussion. All authors read and approved the final manuscript.

Ethics approval and consent to participate

Not applicable.

Consent for publication

Not applicable.

\section{Competing interests}

The authors declare that they have no competing interests.

\section{Publisher's Note}

Springer Nature remains neutral with regard to jurisdictional claims in published maps and institutional affiliations.

Received: 19 June 2018 Accepted: 17 December 2018

Published online: 27 December 2018

\section{References}

1. Cregg JMCJ, Shi J, Higgins DR. Recombinant protein expression in Pichia pastoris. Mol Biotechnol. 2000;16(1):23-52.

2. Gellissen G. Heterologous protein production in methylotrophic yeasts. Appl Microbiol Biotechnol. 2000;54(6):741-50

3. Wang J, Wang $X$, Shi L, Qi F, Zhang $P$, Zhang $Y$, Zhou $X$, Song Z, Cai M. Methanol-independent protein expression by AOX1 promoter with transacting elements engineering and glucose-glycerol-shift induction in Pichia pastoris. Sci Rep. 2017;7:41850.

4. Cereghino JL, Cregg JM. Heterologous protein expression in the methylotrophic yeast Pichia pastoris. FEMS Microbiol Rev. 2000;24(1):45-66.

5. Cereghino GP, Cereghino JL, Ilgen C, Cregg JM. Production of recombinant proteins in fermenter cultures of the yeast Pichia pastoris. Curr Opin Biotechnol. 2002;13(4):329-32.

6. Chen C-C, Wu P-H, Huang C-T, Cheng K-J. A Pichia pastoris fermentation strategy for enhancing the heterologous expression of an Escherichia coli phytase. Enzym Microb Technol. 2004;35(4):315-20.

7. Zhang W, Bevins MA, Plantz BA, Smith LA, Meagher MM. Modeling Pichia pastoris growth on methanol and optimizing the production of a recombinant protein, the heavy-chain fragment $C$ of botulinum neurotoxin, serotype a. Biotechnol Bioeng. 2000;70(1):1-8

8. Sinha J, Plantz BA, Inan M, Meagher MM. Causes of proteolytic degradation of secreted recombinant proteins produced in methylotrophic yeast Pichia pastoris: case study with recombinant ovine interferon-tau. Biotechnol Bioeng. 2005;89(1):102-12.

9. Krasovska OS, Stasyk OG, Nahorny VO, Stasyk OV, Granovski N, Kordium VA Vozianov OF, Sibirny AA. Glucose-induced production of recombinant proteins in Hansenula polymorpha mutants deficient in catabolite repression. Biotechnol Bioeng. 2007;97(4):858-70. 
10. Hilt W, Wolf DH. Stress-induced proteolysis in yeast. Mol Microbiol. 1992 6(17):2437-42

11. Xiao A, Zhou X, Zhou L, Zhang Y. Improvement of cell viability and hirudin production by ascorbic acid in Pichia pastoris fermentation. Appl Microbiol Biotechnol. 2006;72(4):837-44.

12. Prielhofer R, Maurer M, Klein J, Wenger J, Kiziak C, Gasser B, Mattanovich D. Induction without methanol: novel regulated promoters enable high-level expression in Pichia pastoris. Microb Cell Factories. 2013;12:5.

13. Prielhofer R, Reichinger M, Wagner N, Claes K, Kiziak C, Gasser B, Mattanovich D. Superior protein titers in half the fermentation time: promoter and process engineering for the glucose-regulated GTH1 promoter of Pichia pastoris. Biotechnol Bioeng. 2018.

14. Hartner FS, Ruth C, Langenegger D, Johnson SN, Hyka P, Lin-Cereghino GP, LinCereghino J, Kovar K, Cregg JM, Glieder A. Promoter library designed for finetuned gene expression in Pichia pastoris. Nucleic Acids Res. 2008;36(12):e76.

15. Zhang P, Zhang W, Zhou X, Bai P, Cregg JM, Zhang Y. Catabolite repression of Aox in Pichia pastoris is dependent on hexose transporter PpHxt1 and pexophagy. Appl Environ Microbiol. 2010;76(18):6108-18.

16. Shen W, Xue Y, Liu Y, Kong C, Wang X, Huang M, Cai M, Zhou X, Zhang Y, Zhou M. A novel methanol-free Pichia pastoris system for recombinant protein expression. Microb Cell Factories. 2016;15(1):178.

17. Hartner FS, Glieder A. Regulation of methanol utilisation pathway genes in yeasts. Microb Cell Factories. 2006;5:39.

18. Lin-Cereghino GP, Godfrey L, de la Cruz BJ, Johnson S, Khuongsathiene S, Tolstorukov I, Yan M, Lin-Cereghino J, Veenhuis M, Subramani S, et al. Mxr1p, a key regulator of the methanol utilization pathway and peroxisomal genes in Pichia pastoris. Mol Cell Biol. 2006;26(3):883-97.

19. Sahu U, Krishna Rao K, Rangarajan PN. Trm1p, a Zn(II)(2)Cys(6)-type transcription factor, is essential for the transcriptional activation of genes of methanol utilization pathway, in Pichia pastoris. Biochem Biophys Res Commun. 2014:451(1):158-64.

20. Wang X, Wang Q, Wang J, Bai P, Shi L, Shen W, Zhou M, Zhou X, Zhang Y, Cai M. Mit1 transcription factor mediates methanol signaling and regulates the alcohol oxidase 1 (AOX1) promoter in Pichia pastoris. J Biol Chem. 2016; 291(12):6245-61.

21. Wang X, Cai M, Shi L, Wang Q, Zhu J, Wang J, Zhou M, Zhou X, Zhang Y. PpNrg1 is a transcriptional repressor for glucose and glycerol repression of AOX1 promoter in methylotrophic yeast Pichia pastoris. Biotechnol Lett. 2016;38(2):291-8.

22. Liang S, Wang B, Pan L, Ye Y, He M, Han S, Zheng S, Wang X, Lin Y. Comprehensive structural annotation of Pichia pastoris transcriptome and the response to various carbon sources using deep paired-end RNA sequencing. BMC Genomics. 2012;13:738.

23. Vogl T, Sturmberger L, Fauland PC, Hyden P, Fischer JE, Schmid C, Thallinger GG, Geier M, Glieder A. Methanol independent induction in Pichia pastoris by simple derepressed overexpression of single transcription factors. Biotechnol Bioeng. 2018;115(4):1037-50.

24. van Dijk R, Faber KN, Kiel JA, Veenhuis M, van der Klei I. The methylotrophic yeast Hansenula polymorpha: a versatile cell factory. Enzym Microb Technol. 2000;26(9-10):793-800

25. Rodriguez L, Narciandi RE, Roca H, Cremata J, Montesinos R, Rodriguez E, Grillo JM, Muzio V, Herrera LS, Delgado JM. Invertase secretion in Hansenula polymorpha under the AOX1 promoter from Pichia pastoris. Yeast. 1996; 12(9):815-22.

26. Ravin NV, Eldarov MA, Kadnikov W, Beletsky AV, Schneider J, Mardanova ES, Smekalova EM, Zvereva MI, Dontsova OA, Mardanov AV, et al. Genome sequence and analysis of methylotrophic yeast Hansenula polymorpha DL1. BMC Genomics. 2013;14:837.

27. Bawa Z, Routledge SJ, Jamshad M, Clare M, Sarkar D, Dickerson I, Ganzlin M, Poyner DR, Bill RM. Functional recombinant protein is present in the preinduction phases of Pichia pastoris cultures when grown in bioreactors, but not shake-flasks. Microb Cell Factories. 2014;13(1):127.

28. Dragosits M, Stadlmann J, Albiol J, Baumann K, Maurer M, Gasser B, Sauer M, Altmann F, Ferrer P, Mattanovich D. The effect of temperature on the proteome of recombinant Pichia pastoris. J Proteome Res. 2009;8(3):1380-92.

29. Prielhofer R, Cartwright SP, Graf AB, Valli M, Bill RM, Mattanovich D, Gasser B. Pichia pastoris regulates its gene-specific response to different carbon sources at the transcriptional, rather than the translational, level. BMC Genomics. 2015;16:167

30. Camara E, Landes N, Albiol J, Gasser B, Mattanovich D, Ferrer P. Increased dosage of AOX1 promoter-regulated expression cassettes leads to transcription attenuation of the methanol metabolism in Pichia pastoris. Sci Rep. 2017;7:44302.

31. Sahu U, Rangarajan PN. Methanol expression regulator 1 (Mxr1p) is essential for the utilization of amino acids as the sole source of carbon by the methylotrophic yeast, Pichia pastoris. J Biol Chem. 2016;291(39):20588-601.

32. Zhan C, Yang Y, Zhang Z, Li X, Liu X, Bai Z. Transcription factor Mxr1 promotes the expression of Aox 1 by repressing glycerol transporter 1 in Pichia pastoris. FEMS Yeast Res 2017;17(4), fox015.

33. Vogl T, Glieder A. Regulation of Pichia pastoris promoters and its consequences for protein production. New Biotechnol. 2013;30(4):385-404.

34. Vogl T, Sturmberger L, Kickenweiz T, Wasmayer R, Schmid C, Hatzl AM, Gerstmann MA, Pitzer J, Wagner M, Thallinger GG, et al. A toolbox of diverse promoters related to methanol utilization: functionally verified parts for heterologous pathway expression in Pichia pastoris. ACS Synth Biol. 2016; 5(2):172-86.

35. Yu XW, Sun WH, Wang YZ. Xu Y. identification of novel factors enhancing recombinant protein production in multi-copy Komagataella phaffii based on transcriptomic analysis of overexpression effects. Sci Rep. 2017;7(1):16249.

36. Gasser B, Saloheimo M, Rinas U, Dragosits M, Rodriguez-Carmona E, Baumann K, Giuliani M, Parrilli E, Branduardi P, Lang C, et al. Protein folding and conformational stress in microbial cells producing recombinant proteins: a host comparative overview. Microb Cell Factories. 2008;7:11.

37. Damasceno LM, Anderson KA, Ritter G, Cregg JM, Old LJ, Batt CA. Cooverexpression of chaperones for enhanced secretion of a singlechain antibody fragment in Pichia pastoris. Appl Microbiol Biotechnol. 2007:74(2):381-9.

38. Ruth C, Buchetics M, Vidimce V, Kotz D, Naschberger S, Mattanovich D, Pichler H, Gasser B. Pichia pastoris Aft1--a novel transcription factor, enhancing recombinant protein secretion. Microb Cell Factories. 2014;13:120.

39. Guerfal M, Ryckaert S, Jacobs PP, Ameloot P, Van Craenenbroeck K, Derycke $\mathrm{R}$, Callewaert N. The HAC1 gene from Pichia pastoris: characterization and effect of its overexpression on the production of secreted, surface displayed and membrane proteins. Microb Cell Factories. 2010;9:49.

40. Idiris A, Tohda H, Kumagai H, Takegawa K. Engineering of protein secretion in yeast: strategies and impact on protein production. Appl Microbiol Biotechnol. 2010;86(2):403-17.

41. Huang W-T, Tai R, Hseu R-S, Huang C-T. Overexpression and characterization of a thermostable, pH-stable and organic solvent-tolerant Ganoderma fornicatum laccase in Pichia pastoris. Process Biochem. 2011;46(7):1469-74.

42. Stasyk OV, Nazarko TY, Sibirny AA. Methods of plate pexophagy monitoring and positive selection for ATG gene cloning in yeasts. Methods Enzymol. 2008:451:229-39.

Ready to submit your research? Choose BMC and benefit from

- fast, convenient online submission

- thorough peer review by experienced researchers in your field

- rapid publication on acceptance

- support for research data, including large and complex data types

- gold Open Access which fosters wider collaboration and increased citations

- maximum visibility for your research: over $100 \mathrm{M}$ website views per year

At $\mathrm{BMC}$, research is always in progress.

Learn more biomedcentral.com/submission 